\title{
Phase Evolution of Iron Nanoparticles Subjected to Thermal Treatment
}

\author{
K. Brzózka ${ }^{a, *}$, M. Krajewski ${ }^{b}$, T. Szumiata ${ }^{a}$, B. Górka ${ }^{a}$, M. Gawroński $^{a}$, T. Kaczor ${ }^{a}$, \\ W.S. $\operatorname{LIN}^{c}$ AND H.M. LIN ${ }^{c}$ \\ ${ }^{a}$ University of Technology and Humanities, Faculty of Mechanical Engineering, Department of Physics, \\ E. Stasieckiego 54, 26-600 Radom, Poland \\ ${ }^{b}$ Institute of Fundamental Technological Research, Polish Academy of Sciences, \\ A. Pawińskiego 5B, 02-106 Warsaw, Poland \\ ${ }^{c}$ Tatung University, Department of Materials Engineering, Taipei 104, Taiwan, ROC
}

\begin{abstract}
Magnetic nanoparticles based on iron or iron oxides represent an excellent nanomaterial in respect of their fundamental research as well as numerous applications. This work presents a Mössbauer study of the influence of thermal treatment on the structure and phase composition of iron nanoparticles fabricated via a simple reduction reaction. Identification of iron-containing phases has been carried out for the series of samples annealed at different temperatures ranging between $200{ }^{\circ} \mathrm{C}$ and $800^{\circ} \mathrm{C}$. Both crystalline and amorphous iron as well as variety of iron oxides have been detected. It has been proved that the nanoparticles annealed at $500^{\circ} \mathrm{C}$ show superparamagnetic behavior as a result of ultrafine sizes of crystallites of iron oxides that arise in the oxidation processes.
\end{abstract}

DOI: 10.12693/APhysPolA.134.1015

PACS/topics: 61.05.Qr, 61.46.Df, 75.50.Tt, 75.75.Cd, 81.07.-b

\section{Introduction}

Physicochemical properties of nanomaterials differ considerably from their bulk counterparts and depend strongly not only on the kind of substance but also on the shape, dimension, and a way of manufacture [1-4]. This allows to produce materials with diverse prearranged properties, especially when we know the relations between the above mentioned features. There are a large number of reasons for the diversity of nanoparticle properties, between others: increased proportion between atoms situated at the surface and inside the particles along with different coordination number and quite different symmetry of surrounding of surface atoms compared with the interior ones, quantum constrains for electron and phonon wave functions, as well as reduced ratio of particle energy in effective magnetic field to mean energy of its thermal motion which bring on, among others things, superparamagnetic effects. These reasons cause changes in crystal structure, density, viscosity, solubility, thermal properties, mechanical and electrical as well as magnetic characteristics and also chemical activity of nanoparticles in comparison with the corresponding bulk material.

From among many methods of the production of nanomaterials we can distinguish two general categories. The first comprises "top-down" methods which use macroscopic structures as initial systems. All kinds of grinding of the bulk material into small particles

*corresponding author; e-mail: k.brzozka@uthrad.pl (for example milling in electrical mills) [5], severe plastic deformation [6], lithography [7], etc. can be primarily included to this group. "Bottom-up" methods belong to the second class, in which physical or chemical processes are used in order to build, from individual atoms, clusters and afterwards nanoparticles. The latter type allows generally to obtain material more homogeneous in respect of particle sizes but at the same time they can have inhomogeneous structure, for example core-shell nanoparticles. Therefore, many "bottom-up" technologies have been developed in recent years [8-11], between others: sol-gel processing, chemical vapor deposition (CVD), plasma or flame spraying synthesis, laser pyrolysis, atomic or molecular condensation, controlled growing up of nanocrystallites from an amorphous alloy, co-precipitation, electrochemical reduction, reduction reaction of iron salt, etc.

Since nanomaterials are increasingly used in industrial and consumer products, they are the subject of intensive research of the scientists in recent years. Among others, the magnetic nanoparticles based on iron or iron oxides represent an excellent nanomaterial in terms of their fundamental research as well as numerous applications $[12,13]$.

This paper presents a Mössbauer spectroscopy study of the effect of thermal treatment on the microstructure and phase composition of iron nanoparticles fabricated via a simple reduction reaction and next subjected to the annealing at various temperatures. In the earlier reports [14, 15], among others, the results of X-ray diffraction (XRD) and the Raman spectroscopy studies were depicted which gave some information about different phases present in the investigated material, dependently on the annealing temperature. They exhibited 
the presence of the following main phases: bcc iron in as-made nanoparticles as well as bcc iron, magnetite $\left(\mathrm{Fe}_{3} \mathrm{O}_{4}\right)$ and hematite $\left(\alpha-\mathrm{Fe}_{2} \mathrm{O}_{3}\right)$ in various proportions in the annealed samples. The goal of presented study is more detailed - both qualitative and quantitative examination of iron-containing phases, which is enabled by the Mössbauer spectroscopy. Moreover, the analysis of superparamagnetic effects makes possible to draw conclusions about the microstructure of the nanoparticles.

\section{Experimental details}

Iron nanoparticles were synthesized in a chemical reduction of $0.2 \mathrm{ml} 0.5 \mathrm{M}$ hexahydrated iron(III) chloride $\left(\mathrm{FeCl}_{3} \cdot 6 \mathrm{H}_{2} \mathrm{O} ; 98 \%\right.$, Sigma-Aldrich) aqueous solution by $2 \mathrm{ml} 1 \mathrm{M}$ sodium borohydride $\left(\mathrm{NaBH}_{4} ; 98 \%\right.$, SigmaAldrich) aqueous solution and the dissolved oxygen was purged out from the initial solution of iron(III) precursor through a constant argon flow. Afterwards the obtained products of reaction were washed several times with ethanol (99.5\%, Shimakyu's Pure Chemicals Company) and then they were dried in a vacuum oven at $80^{\circ} \mathrm{C}$. Scanning electron microscope (SEM) investigations exhibited that as-prepared nanoparticles are approximately (but not perfectly) spheres. Their diameters vary from 50 to $110 \mathrm{~nm}$, while its mean value comprises between 90 and $100 \mathrm{~nm}$ [14].

As-prepared material was subjected to half hour annealing at different temperatures $T_{\text {ann }}$ (equal to $200^{\circ} \mathrm{C}$, $300^{\circ} \mathrm{C}, 400^{\circ} \mathrm{C}, 500^{\circ} \mathrm{C}, 600^{\circ} \mathrm{C}, 700^{\circ} \mathrm{C}, 800^{\circ} \mathrm{C}$ ) under a slightly oxidizing atmosphere. This procedure made conditions conductive to gradual oxidation of the nanoparticles and, as a result, diversity of phases were created, dependent on the annealing temperature. Specimens for the Mössbauer spectroscopy were prepared in a form of powder layers. For comparison, some experiments were performed for specimens consolidated with glue.

For all the samples, the Mössbauer spectra were collected at room temperature (RT), by the use of ${ }^{57} \mathrm{Co} / \mathrm{Rh}$ source of gamma radiation. Linear changes of the radiation source velocity and vertical direction of the gamma beam were employed. Moreover, for the sample annealed at $500{ }^{\circ} \mathrm{C}$ three Mössbauer measurements were performed in nitric cryostat: at RT and at reduced temperature $\left(-90^{\circ} \mathrm{C}\right.$ and $\left.-181^{\circ} \mathrm{C}\right)$. The same sample was also measured at RT, in an external magnetic field produced by a set of neodymium magnets: (i) perpendicular to the gamma beam, with $B=480.9 \mathrm{mT}$, (ii) longitudinal to the gamma beam, with $B=164.4 \mathrm{mT}$. The Mössbauer spectra, except those that indicated superparamagnetic effects, were analyzed by means of the Normos program using a least-square procedure for fitting lines with the Lorentzian profile.

\section{Results and discussion}

The Mössbauer spectra gathered at RT are presented in Fig. 1 while hyperfine parameters of subspectra determined in the course of the fitting procedure: hyperfine magnetic field (HMF), quadrupole splitting (QS) and central shift (CS) are collected in Table I. The results show that phase composition of the nanoparticles strongly depends on the annealing temperature. In asprepared nanoparticles (Fig. 1a) amorphous iron with possible admixtures of oxygen and other impurities provides the main component with relative spectral area $A=54.8 \%$. It is represented by a smeared Zeeman sextet with the mean hyperfine magnetic field $B=25 \mathrm{~T}$, quadrupole splitting $\Delta=0.08 \mathrm{~mm} / \mathrm{s}$ and central shift $\delta=0.05 \mathrm{~mm} / \mathrm{s}$. The shape of this subspectrum suggests a broad distribution of the hyperfine magnetic field, characteristic of an amorphous phase. This component is accompanied with a sharp sextet characteristic of bcc iron $(B=33.0 \mathrm{~T}, \delta=0.00 \mathrm{~mm} / \mathrm{s}, A=31 \%)$.

Aside from the above mentioned constituents of the spectra, a small contribution $(2-5 \%)$ of a high-field, smeared sextet has been observed, with mean hyperfine magnetic field about $45 \mathrm{~T}$ (for as-prepared nanoparticles) which somewhat decreases with the annealing temperature. This component arises independent of the form of the sample (it occurs also in spectra collected for consolidated specimens) and it is interpreted as traces of strongly distorted oxides that are present at the surface regions of the nanoparticles.

TABLE I

Relative spectral areas (in \%) of the components of Mössbauer spectra collected for nanoparticles before and after heat treatment. Components below 1\% have been omitted. Doublet 1 is attributed to amorphous iron oxides and doublet 2 to superparamagnetic crystalline oxides. The uncertainties amount $1 \%-2 \%$.

\begin{tabular}{l|c|c|c|c|c|c|c}
\hline \hline & \multirow{2}{*}{ As-prepared } & \multicolumn{5}{|c}{$T_{\text {ann }}$} \\
\cline { 3 - 8 } & & $200{ }^{\circ} \mathrm{C}$ & $300{ }^{\circ} \mathrm{C}$ & $400{ }^{\circ} \mathrm{C}$ & $600{ }^{\circ} \mathrm{C}$ & $700^{\circ} \mathrm{C}$ & $800^{\circ} \mathrm{C}$ \\
\hline amorphous iron with admixtures & 54.8 & 29.6 & 23.7 & 10.3 & 3.9 & 4.1 & 4.8 \\
distorted oxides & 5.8 & 1.6 & 2.3 & 4.1 & 0.0 & 0.0 & 0.0 \\
$\alpha-\mathrm{Fe}_{2} \mathrm{O}_{3}$ & 0.0 & 0.0 & 0.0 & 0.7 & 92.4 & 94.6 & 95.3 \\
$\mathrm{Fe}_{3} \mathrm{O}_{4}$ & 0.0 & 1.6 & 0.8 & 4.2 & 2.5 & 1 & 0.0 \\
$\mathrm{bcc-Fe}$ & 29.8 & 25.6 & 22.1 & 12.3 & 0.4 & 0.3 & 0.0 \\
doublet 1 & 0.0 & 22.4 & 18.3 & 5.5 & 0.0 & 0.0 & 0.0 \\
doublet 2 & 7.7 & 17.6 & 29.0 & 61.6 & 0.7 & 0.0 & 0.0 \\
$\varepsilon-\mathrm{Fe}_{2} \mathrm{O}_{3}$ & - & - & 2.3 & 2.0 & - & - & -
\end{tabular}



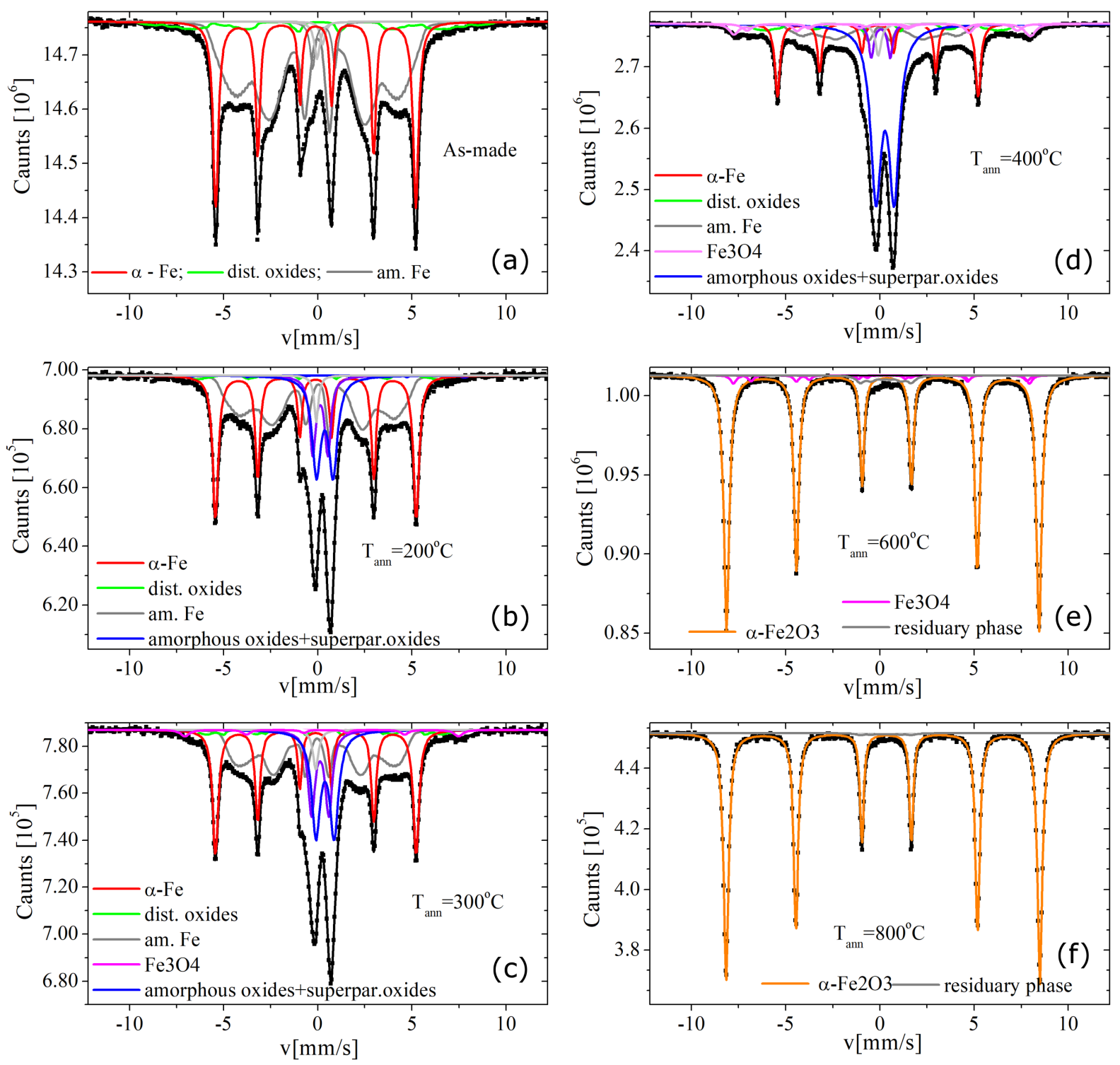

Fig. 1. Mössbauer spectra collected at RT for iron nanoparticles annealed at various temperatures.

On the basis of the obtained results a core-shell model of the as-made nanoparticle structure emerged. In this model, crystalline iron forms the core which is surrounded by a layer of amorphous iron and at its surface small amount of distorted iron oxides occurs. This structure strongly changes owing to gradual oxidation in the course of thermal treatment as described below.

After annealing of the iron nanoparticles at $200^{\circ} \mathrm{C}-$ $400^{\circ} \mathrm{C}$, a central component arises in the spectra in addition to the listed constituents (Fig. 1b-d). It has been fitted mainly by two doublets which are clearly broader than the lines of the sextet belonging to crystalline iron. They are attributed, inter alia, to amorphous oxides (paramagnetic at room temperature) which arise in the nanoparticles in the cost of amorphous iron. A very small (below $1.5 \%$ ) singlet with the isomer shift $0.02 \mathrm{~mm} / \mathrm{s}$, also present in these spectra, is attributed to spuper- paramagnetic iron particles. Crystalline iron oxides, i.e. $\mathrm{Fe}_{3} \mathrm{O}_{4}, \varepsilon-\mathrm{Fe}_{2} \mathrm{O}_{3}, \alpha-\mathrm{Fe}_{2} \mathrm{O}_{3}$, in the form of high-field sextets $(B>45 \mathrm{~T})$ provide only vestigial contribution to the spectra but it increases with rising $T_{\text {ann }}$. A part of them may be in superparamagnetic state because of ultrafine sizes of crystallites, therefore they provide contribution to the set of central doublets. Unfortunately, due to similar parameters of individual doublets they are pure-resolved so we cannot precisely determine the exact relative spectral area of the amorphous oxides and fine crystallites of superparamagnetic oxides. The above explanation is supported by comparison of XRD and the Mössbauer spectroscopy results: XRD spectra show noticeable contribution of magnetite while in the Mössbauer spectra sextets related to this phase amount only about $2 \%-6 \%$. Therefore, the doublets cannot be represented by any other crystalline iron-bearing phase. 

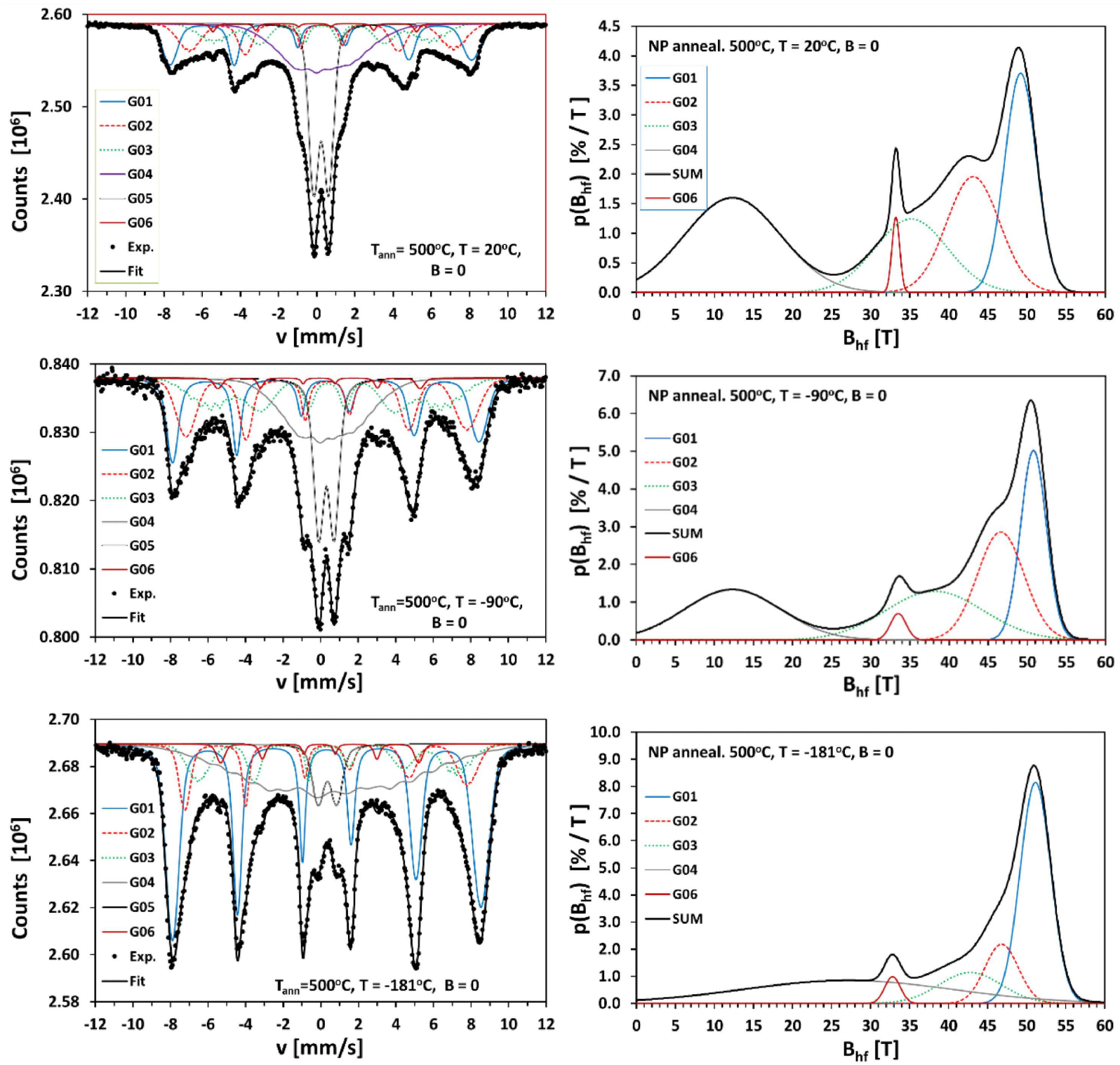

Fig. 2. Mössbauer spectra collected at RT and at lowered temperatures for iron nanoparticles annealed at $500{ }^{\circ} \mathrm{C}($ on the left) and derived distributions of the hyperfine magnetic field (on the right).

As is seen in Figure 1e-f, in the case of nanoparticles annealed at the temperature above $500^{\circ} \mathrm{C}$, hematite has a dominant share to the spectra, although also other phases with lower values of HMF (including magnetite or maghemite $\left.-\gamma-\mathrm{Fe}_{2} \mathrm{O}_{3}\right)$ contribute in a total amount of $5 \%$ even at $T_{\text {ann }}=800^{\circ} \mathrm{C}$.

Room temperature Mössbauer spectrum of the nanoparticles annealed at $500^{\circ} \mathrm{C}$ has a shape different from that previously discussed (Fig. 2a), typical of systems with superparamagnetic relaxations. Although a central component constitutes still significant share of the spectrum, but their contribution makes up only $25 \%$. The other portion of the spectrum takes a shape of several smeared sextets with hyperfine magnetic fields from $10.5 \mathrm{~T}$ to about $44-47 \mathrm{~T}$. The component with the lowest field is presumably a remainder of the amorphous iron with dissolved oxygen (and eventually other indefinite admixtures) that occurs around the core of the particles. The latter component as well as some part of the central doublet can be attributed to crystalline iron oxides in the form of crystallites with nanometric sizes. Due to thermally activated fluctuations of direction of crystallite magnetic moments and some dispersion of their sizes, a superparamagnetic doublet and a wide Zeeman sextet of asymmetric shape coexist in the Mössbauer spectrum. A small sharp sextet related to crystalline bcc Fe is also still observed in the spectrum.

As it is known [16-18], when the thermal energy of a nanocrystallite is comparable to the magnetic anisotropy energy barrier between states corresponding to different directions of the magnetic moment, it causes spin flipping with the mean relaxation time strongly dependent 
on the temperature, the magnetocrystalline anisotropy constant, and the diameter of the nanocrystallite. As a result, energy of a nuclear transition of the Mössbauer isotope is modified, which influences the shape and the position of every line of the Zeeman sextet. When temperature relaxations are fast enough (the relaxation time is smaller than the Larmor precession time), the effective hyperfine magnetic field becomes zero and the nanocrystallites exhibit superparamagnetic behaviour. The following features of the Mössbauer spectra can indicate superparamagnetic relaxations: strong and asymmetric broadening of the sextet lines, coexistence of sextets and a central part of spectrum (doublets, singlets) and reduced values of hyperfine magnetic field determined from the magnetic components in relation to "bulk" values of this quantity [19]. All these features are observed in the spectrum of the nanoparticles annealed at $500^{\circ} \mathrm{C}$.

In order to prove the hypothesis that the significant part of oxides present in the sample takes a shape of fine crystallites, the Mössbauer transmission measurements were performed at temperatures $-90^{\circ} \mathrm{C}$ and $-181^{\circ} \mathrm{C}$ and corresponding Mössbauer spectra are shown in Fig. 2. It is clearly visible that the temperature lowering causes the increase of the spectral area of the Zeeman sextets in the cost of the central doublets and narrowing of the lines inside the sextets, which is a typical of superparamagnetic behaviour.

Although theoretical basis of superparamagnetism is well known, there are no precise and also effective methods of fitting the Mössbauer spectra in the case of fast temperature relaxations of magnetic moments. The reason for this is strong dependence of the relaxation time on several quantities that characterize investigated material and which are not known just enough. Therefore, a very approximate model of fitting has been employed. The spectra collected for the nanoparticles annealed at $500^{\circ} \mathrm{C}$ were fitted using PolMoss program (noncommercial) which utilizes gradient and (parallel) evolutionary optimization algorithms implemented in Solver extension for MS Excel. The broadened sextet was reproduced using several Lorentzian base-line convolution with Gaussian distributions of hyperfine parameters, representing particular phases.

The derived distributions of the HMF are shown on the right side of Fig. 2. The spectra show quite good fit, but interpretation of the particular components of the HMF distribution is ambiguous. The Gaussian distribution with the highest value of hyperfine magnetic field $(G 1)$ is attributed to hematite while the subsequent ones $(G 2, G 3)$ may be related to magnetite and a low-field component $(G 4)$ - to the amorphous remainder. Moreover, a sextet ascribed to bcc iron $(G 6)$ and the central doublet representing superparamagnetic iron oxides $(G 5)$ are present in the spectra.

Evolution of the spectra with the lowering temperature shows a significant increase in the component G1 and simultaneously a marked decrease in the central doublet (G5) (Table II). However, the components G2 and
TABLE II

The values of hyperfine magnetic field and relative spectral areas of the components of Mössbauer spectra (fitted with PolMoss program, see text) collected for nanoparticles annealed at $500{ }^{\circ} \mathrm{C}$.

\begin{tabular}{c|c|c|c|c|c|c}
\hline \hline & \multicolumn{2}{|c|}{$293 \mathrm{~K}$} & \multicolumn{2}{c|}{$183 \mathrm{~K}$} & \multicolumn{2}{c}{$92 \mathrm{~K}$} \\
\cline { 2 - 7 } & $B[\mathrm{~T}]$ & $P[\%]$ & $B[\mathrm{~T}]$ & $P[\%]$ & $B[\mathrm{~T}]$ & $P[\%]$ \\
\hline$G 1$ & 49.2 & 18.8 & 50.8 & 20.7 & 51.1 & 40.1 \\
$G 2$ & 43.1 & 16.6 & 46.6 & 21.4 & 46.8 & 11.7 \\
$G 3$ & 35.2 & 14.5 & 38.1 & 20.1 & 42.7 & 11.4 \\
$G 4$ & 12.3 & 24.7 & 12.3 & 20.6 & 27.1 & 28.7 \\
$G 5$ & 0.0 & 23.8 & 0.0 & 15.3 & 0.0 & 5.6 \\
$G 6$ & 33.2 & 1.6 & 33.5 & 1.8 & 32.8 & 2.5
\end{tabular}

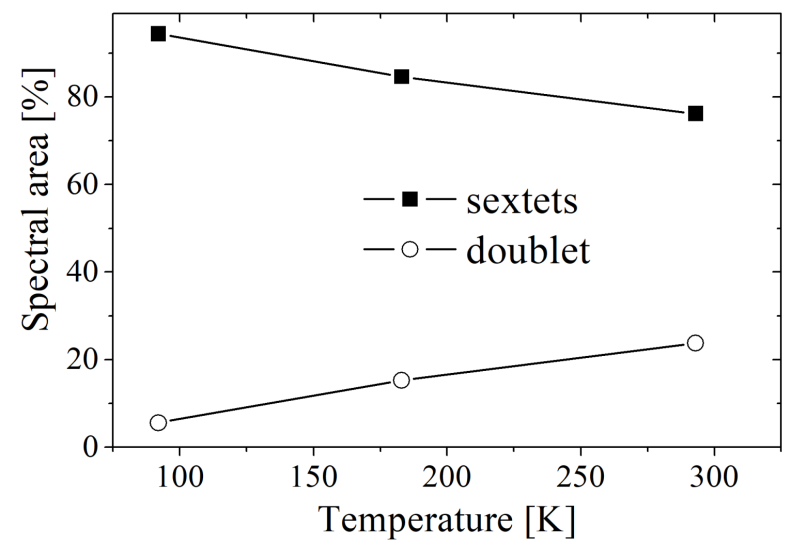

Fig. 3. Temperature evolution of the spectral area of the magnetic and non-magnetic components of the Mössbauer spectra derived for nanoparticles annealed at $500{ }^{\circ} \mathrm{C}$.

G3 are also reduced at the lowest temperature, which is rather unexpected. The contradiction can be removed when we assume that crystallites of the oxides have different diameters. Then, the smallest of them contribute not only to the components $G 1-G 3$, but also to $G 4$, independently of their origin. It causes components $G 1-G 4$ mixing, which explains unusual temperature behaviour of individual spectral areas.

However, there is a general tendency that the total spectral area of the magnetic component of the spectra (i.e. the sum of sextets) increases considerably when the temperature is reduced (Fig. 3). It is worth noticing that the sextet $G 6$ attributed to crystalline iron does not show large line broadening. It follows that iron crystallites are mostly too large to participate in relaxations fast enough to result in superparamagnetic effects.

Therefore, the character of temperature evolution of the spectra collected for the sample annealed at $500{ }^{\circ} \mathrm{C}$ confirms that superparamagnetic oxides, mainly hematite, are prevailing in this case. An additional argument for such a hypothesis is provided by the analysis of spectra collected in an external magnetic field which are shown in Fig. 4. As expected, magnetic field prevents temperature relaxations of direction of magnetic moments. This is manifested in the reduction in the rela- 

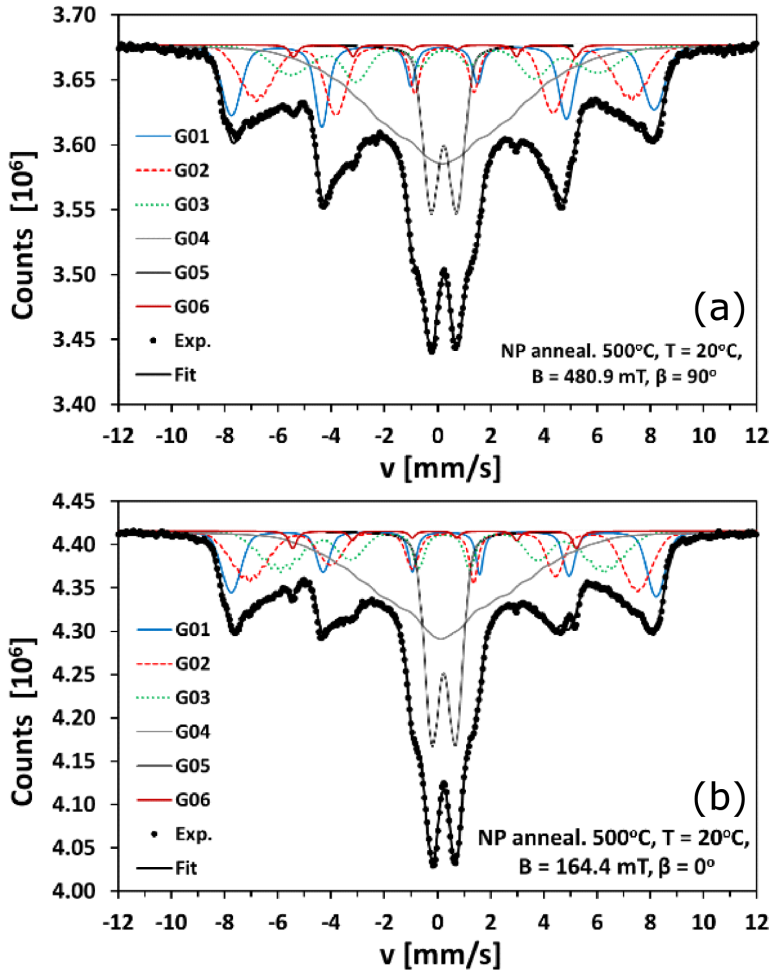

Fig. 4. Mössbauer spectra collected at RT for iron nanoparticles annealed at $500^{\circ} \mathrm{C}$ in external magnetic field perpendicular (a) and longitudinal (b) to the gamma beam.

tive contribution of the non-magnetic components of the spectra, the development of the Zeeman sextets representing oxides as well as the decrease in their linewidth. These features of the Mössbauer spectra are observed for both configurations: with external magnetic field perpendicular and longitudinal to the gamma beam.

\section{Summary and conclusions}

The results of the Mössbauer spectroscopy investigations together with previously-performed SEM, $\mathrm{XRD}$, and Raman spectroscopy studies allowed to determine phase composition of the iron nanoparticles obtained through simple chemical reactions, before and after thermal treatment at different temperatures. It has been stated that as-prepared particles have a core-shell structure with a crystalline iron core surrounded by amorphous iron and traces of iron oxides at the surface. In the case of thermally treated nanoparticles the type of identified phases was strongly dependent on the annealing temperature. It has been exhibited that both $\mathrm{Fe}_{3} \mathrm{O}_{4}, \varepsilon-\mathrm{Fe}_{2} \mathrm{O}_{3}$ and $\alpha-\mathrm{Fe}_{2} \mathrm{O}_{3}$ are formed at different temperature, the latter dominating for annealing temperature $T_{\text {ann }} \geq 600^{\circ} \mathrm{C}$.
The Mössbauer measurements carried out at reduced temperature or in external magnetic field provided arguments for superparamagnetic behavior of iron oxides which are formed at $T_{\mathrm{ann}}=500^{\circ} \mathrm{C}$. This evidences very fine-grain character of the oxidation products, mainly hematite, that arise in these conditions. All this leads to the conclusion that the phase composition of the nanoparticles, including the amount and the kind of iron oxides can be controlled by appropriate heat treatment.

\section{References}

[1] M.B. Bush, Mater. Sci. Eng. A 161, 127 (1993).

[2] Nanophase Materials. Synthesis - Properties Applications Eds. G.C. Hadjipanayis, R.W. Siegel, Springer, Corfu 1994.

[3] D.L. Huber, Small 1, 482 (2005).

[4] D.L. Leslie-Pelecky, R.D. Rieke, Chem. Mater. 8, 1770 (1996).

[5] Chun-Rong Lin, Yuan-Ming Chu, Sheng-Chang Wang, Mater. Lett. 60, 447 (2006).

[6] M. Zehetbauer, R.Z. Valiev, Nanomaterials by Severe Plastic Deformation, Wiley-VCH, 2005.

[7] Q.J. Guo, X.W. Teng, S. Rahman, H. Yang, J. Am. Chem. Soc. 125, 630 (2003).

[8] L.P. Singh, S.K. Bhattacharyya, R. Kumar, G. Mishra, U. Sharma, G. Singh, S. Ahalawat, Adv. Coll. Interface Sci. 214, 17 (2014).

[9] F. Dumitrache, L. Morjan, R. Alexandrescu, V. Ciupina, G. Prodan, L. Voicu, C. Fleaca, L. Albu, M. Savoiu, L. Sandu, E. Popovici, I. Soare, Appl. Surf. Sci. 247, 25 (2005).

[10] S. Veintemillas-Verdaguer, M.P. Morales, C.J. Serna, Mater. Lett. 35, 227 (1998).

[11] Y. Yoshizawa, S. Oguma, K. Yamauchi, J. Appl. Phys. 64, 6044 (1988).

[12] A.S. Teja, P.-Y. Koh, Prog. Cryst. Growth Character. Mater. 55, 22 (2009).

[13] L.L. Vatta, R.D. Sanderson, K.R. Koch, Pure Appl. Chem. 78, 1793 (2006).

[14] M. Krajewski, K. Brzózka, M. Tokarczyk, G. Kowalski, S. Lewińska, A. Ślawska-Waniewska, W. Syuan Lin, H. Ming Lin, J. Magn. Magn. Mater. 458, 46 (2018).

[15] M. Krajewski, W. Syuan Lin, H. Ming Lin, K. Brzózka, S. Lewińska, N. Nedelko, A. ŚlawskaWaniewska, J. Borysiuk, D. Wasik, Beilstein J. Nanotechnol. 6, 1652 (2015).

[16] W.F. Jr. Brown, Phys. Rev. 130, 1677 (1963).

[17] A. Aharoni, Phys. Rev. 135, A447 (1964).

[18] E. Bradford, W. Marshall, Proc. Phys. Soc. 87, 731 (1966).

[19] S. Mørup, Hyperfine Interact. 60, 959 (1990). 